\title{
Les Contraintes Des Coopératives Dans Le Commerce De La Noix De La Cola À Bouaké (Côte d'Ivoire)
}

\author{
Djibli Vincent Dibi \\ Sociologue (Enseignant-Chercheur) \\ Université ALASSANE Ouattara, Bouaké, Côte d'Ivoire
}

Doi: 10.19044/esj.2018.v14n2p68 URL:http://dx.doi.org/10.19044/esj.2018.v14n2p68

\begin{abstract}
This article is an attempt to understand and analyze the difficulties encountered by agricultural cooperatives in the marketing of cola nuts. Despite the considerable capital generated by this sector, many difficulties are hampering the agricultural sector, which results in a sharp decline in the profits of cooperatives and producers. For the members, this is due to the lack or little commitment of the Ivorian State to revitalize this promising sector as is the case with the coffee-cocoa pair. In fact, the fact that foreign traders have direct access to growers in their production areas without going through a central structure, in particular the wholesale marketBouake, weakens domestic traders. Similarly, this situation prevents the Ivorian export circuit of Cola from having a substantial tonnage and allowing the entry of foreign currency into the country. All this contributes to devalue the label of the cola nut produced in Cote d'Ivoire.
\end{abstract}

Keywords : Constraints, cooperatives, cola nuts, wholesale market, marketing

\section{Résumé}

Cet article est un essai à la compréhension et à l'analyse des difficultés que rencontrent les coopératives agricoles dans la commercialisation de la noix de cola. Malgré les importants capitaux générés par cette filière, de nombreuses difficultés minent ce secteur agricole qui engendrent une forte baisse des bénéfices des coopératives et producteurs. Pour les sociétaires, cela est dû au manque ou peu d'engagement de l'État ivoirien à redynamiser cette filière prometteuse comme c'est le cas avec le binôme café-cacao. En réalité, le fait que les commerçants étrangers ont directement accès aux planteurs dans leurs zones de production sans passer par une structure centrale notamment le Marché de Gros de Bouaké, affaiblit les commerçants nationaux. De même, cette situation empêche le circuit d'exportation ivoirien de Cola d'avoir un 
tonnage conséquent et permettant l'entrée des devises dans le pays. Tout cela concourt à dévaloriser le label de la noix de cola produite en Côte d'Ivoire.

Mots clés: Contraintes, coopératives, noix de cola, marché de gros, commercialisation

\section{Introduction}

Dès son accession à l'indépendance, la Côte d'Ivoire a orienté sa politique économique sur l'agriculture. Ainsi, la crise économique des années 80 a conduit le pays à modifier sa politique en libéralisant son économie agricole et cela à la faveur des Programmes d'Ajustement Structurel (PAS). L'agriculture ivoirienne s'est organisée pour les principales productions en activité de recherche, d'encadrement des producteurs et de commercialisation de la production du pays. Cette stratégie qui a permis de développer certaines filières telles que le cacao, le café, le coton, l'hévéa etc., n'a pu être étendue à des produits tels que la noix de cola qui avait cependant une intense activité informelle.

En Côte d'Ivoire, l'importance de la filière cola n'est plus à démontrer, vu qu'en termes de production, le pays fournit à lui seul chaque année 80 mille tonnes de noix fraiches (CNRA, 2012). Cette production engendre un chiffre d'affaires estimé à 100 milliards de FCFA liés à la commercialisation de ce produit (INADES-Formation, 2012). Quant à la production, la noix cola est cultivée sur presque l'ensemble du territoire avec des superficies allant de 1 à 15 hectares. Cela montre que cette culture n'est plus un simple produit de la cueillette mais une activité de production. Pour la commercialisation proprement dite, la Côte d'Ivoire est le premier exportateur de noix de cola dans le monde et dispose de deux grands centres de transit et de commercialisation qui sont le Marché de Gros de Bouaké (MGB) et la ville d'Anyama. De plus, au niveau des acteurs, sur les 1127 commerçants grossistes identifiés au MGB, 748 opèrent dans la filière en tant que acheteurs, collecteurs et exportateurs soit environ les $2 / 3$ des acteurs du marché (Aloko, 2000).

En fait, l'activité commerciale de la filière cola prend son sens du fait de la construction du MGB en 1998. Et pour la seule année de 1999 les échanges avoisinaient les 19 mille tonnes de cola (Aloko, 2000). Pour les 18604 tonnes vendues en 1999, le commerce de la noix de cola a réalisé un chiffre d'affaires de près de 15 milliards de F CFA. Ces chiffres d'affaires ont été réalisés grâce au dynamisme des coopératives agricoles d'avant la crise militaro-politique de 2002. Aujourd'hui, le négoce du cola occupe une place importante dans l'économie ivoirienne (un chiffre d'affaire exprimé à plus de 
100 Milliard de FCFA, soit 150 Millions d'Euros) $)^{2}$ car c'est l'un des produits d'exportation par excellence du pays.

Bien que la commercialisation de la noix de cola soit une activité très importante pour l'économie ivoirienne et particulièrement pour le marché de gros de Bouaké, cette filière est minée par une faiblesse de la réglementation étatique. Ce manque d'implication de l'État à l'égard de cette filière cola, est une injustice du fait que les autres filières agricoles telles que celles du cafécacao, du coton et de l'anacarde bénéficient d'une attention particulière de l'État. Cela a pour conséquence la mauvaise organisation de la filière cola. Celle-ci s'est accentuée avec la crise militaro-politique de 2002 et la crise postélectorale de 2011 qui ont sérieusement affaibli la capacité et le dynamisme des coopératives de cette filière. Aujourd'hui, les coopératives doivent faire face à de réelles difficultés pour assurer leur survie dans cette filière.

En effet, ces problèmes n'ont pas laissé indifférents les observateurs et les chercheurs qui ont produit à ce sujet de nombreuses publications : (Aloko, 2000) ; (Kouame, 2015) ; (Njonga, 2009) ; (FIRCA, 2013).

Mais les dimensions socio-économiques qui retiennent notre attention ici sont celles qui sont en rapport avec les difficultés qui minent la filière cola représentant une entrave au bon fonctionnement des coopératives. Ces difficultés ont fait l'objet de tentatives de résolution qui n'ont pas abouti à une issue heureuse. De telles situations ont ralenti les activités et la capacité de commercialisation de la noix de cola des coopératives agricoles du MGB.

Alors, quelles sont les contraintes que les coopératives agricoles subissent dans la commercialisation de la noix de cola au Marché de Gros ?

L'objectif de cet article est d'identifier et d'analyser l'ensemble des contraintes qui minent le bon fonctionnement des coopératives agricoles dans la commercialisation de la noix de cola, gage de l'existence d'une filière dynamique pour une économie prospère.

La thèse soutenue est la suivante : les contraintes que rencontrent les coopératives agricoles dans la commercialisation de la noix de cola, filière peu organisée, obligent les coopératives et les acteurs de ladite filière à opter pour de nouvelles stratégies commerciales leur permettant d'assurer la survie du commerce de cola comme une activité socio-économique importante. Cependant la crise militaro politique 2002 est venue aggraver cette situation précaire des coopératives et par ricochet celle de la filière. Car les opérateurs économiques de la sous-région qui étaient contraints à se ravitailler au MGB avant la crise, se dirigent directement vers des paysans dans les zones de production. Après la crise, les coopératives du marché de gros ne sont plus les

\footnotetext{
${ }^{2} \mathrm{La}$ cola ne nourrit plus son homme |Slate Afrique. URL: http : //www.slateafrique.com/30671/, Côte-d-Ivoire-noix-de-cola-économie, Consulté le 21/11/2016.
} 
seuls les acteurs à se ravitailler auprès des paysans dans les différentes zones de production du pays.

Les théories du choix rationnel et de la résilience seront sollicitées ici pour en donner l'éclairage, parce qu'elles permettent de mieux maitriser et de comprendre les raisons et les comportements des acteurs face à l'ensemble contraintes qu'ils rencontrent.

Les données à analyser sont essentiellement recueillies au moyen de méthodes qualitatives dans un premier temps. Nous avons privilégié les entrevues semi-structurées auprès des acteurs des coopératives de cola du MGB. Ces parcours et trajectoires recueillis nous ont permis de repérer les contraintes, les stratégies et les choix éventuels des acteurs. Ces outils qualitatifs ont été complétés par une enquête quantitative axée sur un questionnaire et une revue documentaire. Ce sondage avait pour objectif de vérifier certaines hypothèses à travers les variables que nous avons fait ressortir, après l'analyse des entretiens qualitatifs, comme étant les plus significatives pour la compréhension des raisons et des comportements des acteurs, des coopératives agricoles de notre étude. Ce sondage a été réalisé auprès des sociétaires, sinon des instances dirigeantes du MGB et des coopératives. Ces enquêtes ont lieu dans la localité de Bouaké. En tout, 35 personnes ont été enquêtées.

Ainsi dans un premier temps, nous présenterons la place de la filière cola au MGB. Cela nous permettra dans un second moment d'identifier et d'analyser les contraintes et difficultés de production, économiques, administratives, organisationnelles, législatives, infrastructurelles, sociales et humaines liées à la commercialisation de la noix de cola. Enfin, dans un troisième temps, nous essaierons de montrer les stratégies et moyens utilisées par les coopératives agricoles pour la commercialisation de la noix de cola.

\section{Présentation De La Filière}

\section{Organisation de la filière au plan national}

La Filière Cola reste confrontée à une absence de professionnalisation des acteurs. Nonobstant cet état de fait, plusieurs organisations opèrent dans la filière. Depuis Octobre 2011, est née une faitière de ces associations dénommée, la Fédération Nationale des Professionnels de la filière Cola de Côte d'Ivoire (FENAPROCOCI).

En 2015, la filière cola en Côte d'Ivoire a décidé de mieux s'organiser afin de booster ces opérations de production, de collecte, de stockage, de transformation, de commercialisation et de rentabilité. En effet, les acteurs de la filière cola se donnent les moyens de redynamiser les activités de ce secteur. Cela s'est traduit par la création de deux sociétés coopératives. Ainsi, l'Union des sociétés coopératives des producteurs de cola de Côte d'Ivoire (Unaproco- 
Ci), et l'Union des sociétés coopératives des exportateurs et commerçants de cola de Côte d'Ivoire (Uscopex-Ci) ont vu le jour en 2015.

\section{Importance de la noix de cola}

Elle trouve son fondement dans l'usage que la population ivoirienne fait de la noix de cola. En effet, ce produit est au centre de plusieurs cérémonies en Afrique de l'Ouest notamment en Côte d'Ivoire. La noix de cola est omniprésente dans la vie sociale et culturelle de la population. D'abord au niveau culturel, cette noix participe aux évènements heureux et malheureux tels que l'annonce de la venue d'un enfant au monde, lors de son baptême et scelle l'union des futurs mariés. Sans oublier que la noix est utilisée pour honorer les défunts pendant les funérailles dans certaines cultures ivoiriennes (Aloko, 2000). De plus, la noix de cola est parfois offerte en guise d'aumône pour conjurer le mauvais sort ou pour attirer la chance ou comme cadeau aux grands-parents et amis ${ }^{3}$. Pour ce qui est du niveau social, la noix de cola fait usage de remède. Du fait de sa teneur en caféine, elle se révèle être un stimulant nerveux et physique augmentant la résistance du consommateur à la fatigue. Cette noix se présente ainsi comme un aphrodisiaque et recèle des vertus diurétiques et cardiotoniques.

L'importance de la commercialisation de la noix de cola ressort dans les entretiens que nous avons menés avec nos enquêtés, nous ont permis de constater que parmi les neuf filières existantes au MGB, Le secteur du cola est le secteur le plus productif. En effet, malgré les perturbations qu'elle connait, la filière cola produit plus de la moitié des recettes du marché de gros.

"La filière cola est la plus sinistré de ce marché mais malgré tout ça nous faisons les $90 \%$ du marché de gros. ", M. CAB (secrétaire général de la coopérative Sipa-ci).

Malgré son importance, la commercialisation de la noix de cola rencontre diverses formes de difficultés dans la pratique.

\section{Les contraintes dans la commercialisation \\ La concurrence déloyale}

Les entretiens que nous avons menés avec nos enquêtés, nous ont permis de constater qu'il existe une forte concurrence déloyale entre les zones de production et le MGB. En effet, les zones de production ne ravitaillent plus convenablement le MGB, elles préfèrent faire fi du MGB pour commercialiser directement leur cola aux exportateurs. Cette situation se perçoit à travers les propos de M. DAS :

\footnotetext{
${ }^{3}$ Cette pratique a surtout lieu dans les sociétés soudano-sahéliennes à dominance musulmane (malinké, senoufo etc...) en Côte d'Ivoire
} 
"Quand les gens chargent la cola à Anyama, Soubré, Danané, il ne passent plus ici, ils vont directement livrer la cola au Mali, Burkina et autre. Et cela, ne nous arrange pas, ça diminue nos clients et ça fait qu'on ne vend plus normalement. » (Commerçant grossiste).

Aussi, les commerçants grossistes ne se ravitaillent plus au MGB et vont directement chercher leur cola dans les zones de production sans payer de taxe, ce qui leur revient encore moins chère par rapport au MGB : "A cause des taxes de 550fr par panier les gens ne viennent plus prendre cola avec nous, il nous laisse pour aller directement à Anyama et au champ parce que la bas c'est moins chère, y'a pas de taxe à payer, et quand c'est comme ça, ça nous arrange pas. », M. SGY (membre de SIPA-CI).

En fait, le manque ou l'insuffisante de l'implication de l'État ivoirien dans la fixation du prix d'achat et de vente de la noix de cola expose les commerçants entre eux à une concurrence exagérée et incontrôlée. Ainsi, cette concurrence tire son origine d'une part dans la volonté de contrôler le marché à travers le prix d'achat dans les zones de production et au MGB entre commerçants et d'autre part, dans la vente de la cola sur les marchés étrangers (Mali, Niger, Burkina...). Concernant ces deux aspects, sur les 24 commerçants, 21 répondants soit $87,5 \%$ ont reconnu ces éléments comme facteurs motivants de la concurrence inégale entre eux dans la conduite de leurs activités. Pour avoir plus de produits dans les zones de production les commerçants se livrent à des pratiques parfois malsaines pour obtenir le meilleur produit à bon prix. Ainsi selon K.M ${ }^{4}$. de SIPACI :

"Il y a des commerçants, parce qu'ils veulent avoir beaucoup de produits peuvent augmenter les prix d'achat dans la brousse et souvent ils vont jusqu'à acheter les produits que des commerçants ont commandé avec les planteurs. Et comme eux (les planteurs), ils cherchent à avoir plus de profit, ils vendent et quand tu arrives sur le terrain, ils te remboursent ton argent parce qu'ils ont vendu à un autre que tu connais, c'est comme ça ici!"»

Tableau et graphique 1 : répartition des commerçants sur la concurrence dans la filière cola

\begin{tabular}{|c|c|c|}
\hline & Nombre d'individus & Fréquence \\
\hline Réponse & 21 & $87,5 \%$ \\
\hline Oui & 3 & $12,5 \%$ \\
\hline Non & 24 & $100 \%$ \\
\hline
\end{tabular}

4 Personne interviewé lors de l'enquête 


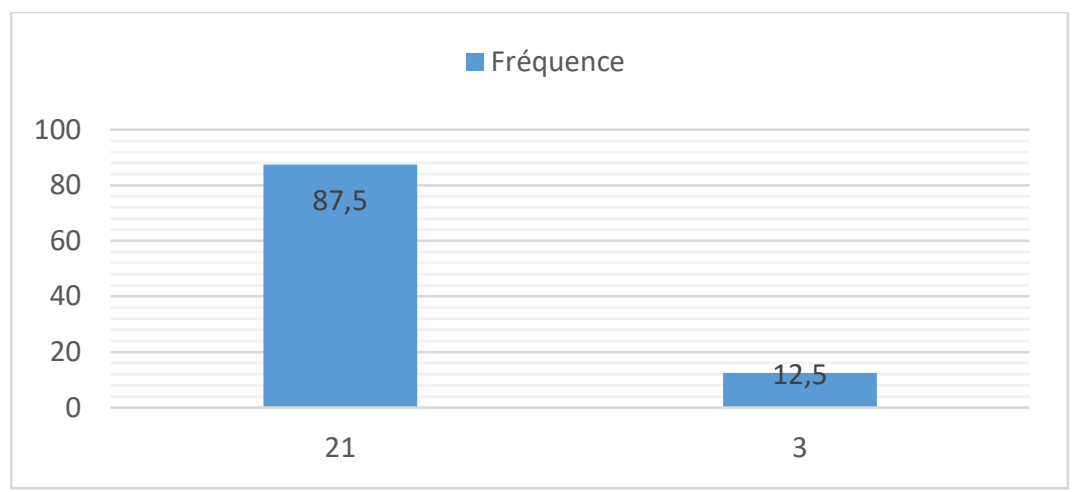

Source : nous investigations de terrain 2017

\section{L'accès au crédit de démarrage et de fonctionnement par les commerçants}

Le crédit de démarrage et de fonctionnement sont des éléments importants dans le commerce des produits agricoles et surtout dans la filière cola. Mais sur les 24 commerçants seulement 16 soit 66,66 \% d'entre eux ont demandé un crédit dans une banque ou micro finance et les 8 soit 33,33\% n'ont pas eu recours au crédit de démarrage et de fonctionnement. Parmi ceux qui ont demandé le crédit pour leur commerce de cola aucun d'eux n'a eu de satisfaction. Pour justifier ce refus de la part des institutions de crédit Madame K. A. ${ }^{5}$ de la BHCI a expliqué que :

«Ce sont des produits (agricoles) dont l'activité n'est pas constante notamment les prix alors que notre banque est une structure commerciale et qui recherche du profit donc nous voulons avoir de l'assurance par rapport aux risques que nous prenons dans l'octroi de crédit et le financement des activités de nos clients. Mais au niveau de la noix de cola nous ne maitrisons rien au sujet de cette activité donc nous $n$ 'accordons pas de crédits dans ce secteur et en général dans le commerce des produits agricoles ici à Bouaké. »

Repartions des commerçants par rapport à la demande de crédit

\begin{tabular}{|c|c|c|}
\hline $\begin{array}{c}\text { Demande } \\
\text { de crédits }\end{array}$ & $\begin{array}{c}\text { Nombre } \\
\text { d'individus }\end{array}$ & Fréquence \\
\hline Oui & 8 & $33,33 \%$ \\
\hline Non & 16 & $66,66 \%$ \\
\hline Total & 24 & $100 \%$ \\
\hline
\end{tabular}

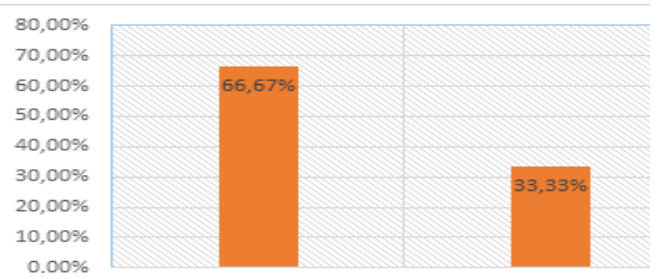

Source : nous investigations de terrain 2017

5 Personne interviewée lors de l'enquête 
Ces propos mettent en exergue la méfiance des institutions financières par rapport au commerce de la noix de cola et surtout de sa rentabilité pour les coopératives. En outre, concernant les 16 personnes qui ont demandé un prêt, six (06) ont soumis un dossier de prêt dont le montant se situe entre 200.000 FCFA et 500.000FCFA. Quant au dix (10) autres restant ont sollicité des prêts dont le montant commerce à partir de 500.000 FCFA.

\section{La corruption dans le secteur}

Les tracasseries routières à répétition que les transporteurs et les commerçants de la coopérative SIPA-CI subissent dans la commercialisation de la noix de cola les amènent ceux-ci à adopter des comportements visant à instaurer un climat frauduleux dans le secteur du cola et cela se fait avec la complicité des forces de l'ordre, qui sont eux les premiers responsables de ces actes.

"Quand j'arrive chez les douaniers et les eaux et forêt, on discute et on tombe d'accord sur un prix, je paye rapidement et je $m$ 'en vais pour ne pas que mon chargement brûle sous le soleil. », M. BDL ${ }^{6}$ (transporteur).

Selon les commerçants de la coopérative, les principaux acteurs de cette pratique sont respectivement les agents des eaux et forêts, les douaniers, la police, les militaires et les gendarmes parfois. Cette pratique a lieu au niveau des barrages routiers à l'intérieur du pays et aux frontières extérieurs. Quant aux motifs du racket, les commerçants estiment qu'il n'existe pas un fondement valable qui justifie ces agissements de la part des forces de l'ordre. Concernant les agents des eaux et forêts, selon les propos de K.C. ${ }^{7}$ :

"Pour racketter; les Eaux et Forêts disent que la noix de cola est un produit de cueillette donc c'est un produit forestier. Lorsqu'on dit que ne s'est pas un produit forestier, ils nous disent que le rotin ou les feuilles qu'on a pris pour emballer sont des produits forestiers et demandent parfois, qu'on décharge le camion sur la route et comme ça on paie entre $5000 \mathrm{f}$ cfa et $10000 \mathrm{f}$ cfa par barrages selon la quantité du produit. »

Ces propos résument clairement la situation du racket sur les routes et surtout la commercialisation de la cola. Cette pratique vient augmenter les nombreuses charges engendrées par l'achat et le transport de ce produit et hautement périssable.

6 Personne interviewée lors de l'enquête

7 Personne interviewée lors de l'enquête 


\section{La dévalorisation du cola ivoirien}

Les différentes stratégies mis en place par les commerçants pour échapper aux difficultés liées à la commercialisation de la noix de cola laisse apparaître une dévalorisation de ce produit. Les commerçants voulant contourner ces difficultés les rendent donc vulnérable face aux opérateurs étrangers. En effet, le financement de l'activité de commercialisation de la noix de cola est essentiellement réalisé par les opérateurs étrangers vivant à l'extérieur du pays. Cette situation fait que le cola ivoirien n'est pas reconnu en Europe car elle perd son label au profit de celle d'autre pays tel que le Mali et le Nigeria. Cette situation se perçoit à travers les propos de $\mathrm{M}$. $\mathrm{CAB}^{8}$ :

"Lorsqu'on vend la noix de cola, arrivé dans les autre pays comme le Nigéria, ils prennent notre cola ils mettent leur étiquette dessus pour vendre dans les pays européens, ce qui fait qu'aujourd'hui, le Nigéria est l'un de plus grand exportateur de cola au monde. "), (secrétaire général de la coopérative Sipaci).

Cette situation traduit le manque de suivi du secteur du cola non seulement par les acteurs de la filière mais aussi le manque de l'implication de l'État ivoirien.

\section{L'absence de contractualisation}

Dans le souci de vendre leur cola à tout prix, les commençants ne se soucient pas de prendre des mesures préventives contre d'éventuels dommages qui pourraient survenir lors des échanges commerciaux. Ainsi, cette situation les amène donc à réaliser de grosses pertes au cours du transport de la noix de cola vers d'autre destination, notamment dans les pays de la sousrégion. Les propos de $\mathrm{M}$. K. ${ }^{9}$ un enquêté nous permettent de comprendre cette situation :

"Si y'a pas de contrat on ne prend pas de mesures préventives contre quoi que ce soit. Par exemple monsieur Y à la cola et il veut vendre à monsieur $X$, maintenant monsieur $X$ va lui dire moi je veux vendre ta cola, mais il ne va pas lui dire je veux payer ta cola hein, donc toi tu prends ta cola tu lui envoies avec tous les risques. Et pendant que lui il est entrain de vendre où toi tu vas gagner 500fr de bénéfice lui il va gagner $2000 f r$ de bénéfice, et lui non seulement il gagne plus

\footnotetext{
${ }^{8}$ Personne interviewée lors de l'enquête

${ }^{9}$ Personne interviewée lors de l'enquête
} 
que toi mais tous ce qui est perte et sinistre relève de

toi." ")

Il rajoute ensuite :

" Je vous donne un exemple concret, les gars ont envoyé 4 camions au Niger, le monsieur a reçu la cola, ce qui est sûre il a vendu une partie et puis la noix de cola a cramée à cause de la chaleur; on dit vous avez envoyé 4 camions les 4 camions ont brûlés. Donc il y a un problème de contractualisation. Pas de protection, pas d'assurance. ",, M. K (contrôleur de gestion du MGB).

\section{La multiplicité des intervenants publics dans le commerce de la noix de cola}

La commercialisation de la noix de cola fait intervenir plusieurs acteurs notamment les acteurs publics. Ainsi au niveau de Bouaké le commerce de la noix de cola fait appel aux services suivants : OCPV, la douane, Direction régionale de l'agriculture, la direction régionale du ministère de l'industrie, le marché de gros, la chambre de l'industrie et de commerce. L'ensemble de ces structures chacun à son niveau et selon sa compétence agissent sur la commercialisation de la noix de cola en collectant des taxes sur la chaine de commerciale. Par ailleurs, les commerçants doivent obtenir des documents administratifs avant le changement et la sortie des produits hors de la Côte d'Ivoire. Ainsi dès lors qu'une structure n'a pas produit de document le chargement ne peut sortir du marché.

\section{Les compétences techniques et les moyens de contrôle de la qualité des produits}

Elle concerne les structures du ministère de l'agriculture par le biais de la Direction Régionale de l'Agriculture. En réalité, cette structure a pour mission de contrôler la qualité des toutes les noix de cola qui sortent et entrent au MGB. Mais, ces agents en charge n'ont pas de formation requise et la maitrise des procédés techniques comme l'attestent les propos du chef de service de contrôle qualité. Selon lui :

«Il n'y a pas de normes de qualités définis pour la noix

de cola. Donc le contrôle que nous faisons est subjectif.

En plus nous n'avons pas des moyens techniques ou

instruments de mesure pour le contrôle. Donc nous faisons avec, comme on le dit. "

Ces propos montrent clairement les difficultés auxquelles les agents de contrôle font face sur le terrain et les conditions de travail de ces agents.

\section{Les conflits d'attributions des structures administratives publiques}


Les conflits des structures publiques sont liés à leurs attributions respectives. Ainsi la commercialisation de la noix de cola nécessite l'intervention de plusieurs structures déconcentrées de l'État. Cependant ces structures se disputent parfois leurs différentes prérogatives dans leur implication dans le commerce de la noix de cola. Ainsi selon les informations recueillies sur le terrain il y a plus de 4 structures qui y interviennent. Mais le conflit est le plus ouvert entre les services du ministère de l'agriculture et ceux de l'industrie. En effet, selon un responsable :

«Il y a les services du ministère de l'industrie qui disent que ce sont eux qui doivent intervenir et que c'est à eux que revient la filière, alors que la cola n'est pas exploiter industriellement donc tu vois qu'Il ne doit même pas prélever quoi que ce soit sur la cola, mais ils le font. Donc nous avons le plus souvent du mal à nous comprendre dans cette situation. »

Retenons que ces propos élucident le conflit voilé entre les structures publiques dans la commercialisation de la noix de cola à Bouaké.

\section{Les stratégies}

\section{La délocalisation des commerçants vers d'autres sites}

Notre passage sur le terrain nous a permis d'observer chez les commerçants cette forte tendance à quitter le MGB pour s'installer sur d'autres sites. En effet, face aux redevances des magasins sont trop élevées, des taxes au niveau du marché à n'en point finir et les tracasseries routières, certains commerçants ont décidé de s'implanter définitivement au niveau des zones de production. Ainsi, à partir de ces zones de production, ils rallient la marchandise vers les marchés d'écoulement au niveau des pays qui sont les principaux clients du MGB, sans transiter par le marché de gros. Les propos d'un enquêté nous permet de s'en convaincre :

"Les magasins sont chères, on nous fatigue en route et les taxes au marché ici sont trop élevées et quand tu arrives marché ici tu dois payer au pont bascule encore. Tous nous fatigue, c'est pourquoi d'autre commerçants fuient pour aller rester à Anyama et ne reviennent plus », M. MDS (commerçant de SIPA-CI).

Ainsi, la principale contrainte selon les commerçants des coopératives de la filière de la noix de cola est le financement de l'activité de façon générale. Sur 24 commerçants de SIPA-CI interrogés, la totalité soit les $100 \%$ ont indiqué que le financement est la plus grande difficulté. En dehors de cela il y a aussi les conditions difficiles d'accès des zones de production de la noix de cola. À ce niveau sur les 24 commerçants 10 représentants $41,70 \%$ ont recours 
à l'aide familiale pour financer leur activité. À ce sujet sur les 24 personnes 22 d'entre eux soit $91,70 \%$ ont constitué leurs fonds de commerce par cette pratique. La majorité des commerçants trouvent les montants de ce type insuffisant pour à bien leur commerce. Ainsi l'ensemble de nos enquêtés estiment que les montants réunis sont insuffisants pour générer des bénéfices élevés. La faiblesse des ressources fait que les commerçants sont obligés d'acheter de petits quantités de cola ce qui a pour conséquence directe d'engendrer des revenus faibles tirés de leur commerce.

\section{Conclusion}

Au terme de notre étude, nous pouvons relever la confirmation de nos hypothèses. En effet, la ville de Bouaké à travers le marché de gros constitue un espace de stockage des produits en provenance des zones de production. En plus, le prix aux producteurs sont fixés en fonction des informations provenant des marchés des pays tels que le Mali, Guinée, Burkina Faso et le Niger par le marché de gros. En outre, le marché de gros a pour fonction d'assurer l'approvisionnement des autres marchés intérieurs, les détaillants de la ville même parfois Anyama et les villes autour de Bouaké.

En somme, nous avons observé que les stratégies adoptées par la coopérative Sipa-ci pour s'approvisionner et commercialiser la noix de cola au MGB présente d'énormes conséquences qui tendent à fragiliser de façon considérable le secteur de la filière cola tant au niveau local qu'international. Cependant, vu rôle que joue la noix de cola dans l'industrie et l'importance économique de la filière cola en Côte d'Ivoire, chaque acteur adopte des logiques et comportements sociaux pour s'approvisionner à moindre coût. Ce en vue de réaliser d'importants bénéficies lors de la commercialisation de ce produit. $\mathrm{Au}$ vu de tout ce qui précède, les différents opérateurs doivent davantage unir leurs forces à travers la coopérative pour faire bénéficier à la fois les acteurs pris individuellement et leurs coopératives.

\section{References:}

1. ALOKO-N'GUESSAN J., 2000, Cola, espace et sociétés : étude de géographie sociale et culturelle de la filière de la cola au marché de Gros de Bouaké, Université de Cocody, Abidjan (Côte d'Ivoire).

2. DABIE DÉSIRE AXEL NASSA., 2010, Approvisionnement en produit vivriers d'une ville secondaire ivoirienne à l'ère de la mondialisation : l'exemple de Divo, <halshs-00476299>.

3. EGNONTO M. KOFFI- TESSIO., et al., 2007, Les Marges de Commercialisation et L'équité du Commerce des Produits Alimentaires au Togo, AAAE Conference Proceding.

4. AGRICI.NET [www.Agrici.net]., 23 mars 2016, « La filière cola en pleine souffrance dans la région du Gbêkê ». 
5. AGRI BI-MENSUEL., Le parlement adopte la loi d'Orientation Agricole de Côte d'Ivoire(LOACI) dite "Loi Sangafowa"», N02Jeudi 16 juillet 2015.

6. ALFRED KOUAME., 29 juillet 2015, Fratmat.info (Abidjan), « Côte d'Ivoire : Filière cola - Producteurs et exportateurs créent deux sociétés coopératives pour être dynamiques ».

7. BERNARD NJONGA (dir.), 14 Décembre 2009, La voix du paysan, «Cola : Transactions commerciales : aperçu sur le marché de la cola au Cameroun ».

8. FIRCA, « acte 12 : la filière cola », présentation de la filière cola ; numéro 12 du 4 trimestre 2013.

9. LE PARTI ÉCOLOGIQUE IVOIRIEN, Commercialisation des produits vivriers : le marché de gros de Bouaké se meurt, consulté le 15/03/2016. 